\title{
EXISTENCE AND CONTINUOUS DEPENDENCE FOR A CLASS OF NONLINEAR NEUTRAL- DIFFERENTIAL EQUATIONS
}

\author{
L. J. GRIMM ${ }^{1}$
}

Abstract. This paper presents existence, uniqueness, and continuous dependence theorems for solutions of initial-value problems for neutral-differential equations of the form

$$
x^{\prime}(t)=f\left(t, x(t), x(g(t, x)), x^{\prime}(h(t, x))\right), \quad x(0)=x_{0},
$$

where $f, g$, and $h$ are continuous functions with $g\left(0, x_{0}\right)=h\left(0, x_{0}\right)$ $=0$. The existence of a continuous solution of the functional equation $z(t)=f(t, z(h(t)))$ is proved as a corollary.

1. Introduction. In this paper we consider the initial-value problem (IVP) for the functional-differential equation of neutral type

$$
x^{\prime}(t)=f\left(t, x(t), x(g(t, x(t))), x^{\prime}(h(t, x(t)))\right),
$$

with the initial condition

$$
\text { (2a) } x(0)=x_{0} \text {. }
$$

Here $f(t, x, y, z), g(t, x)$ and $h(t, x)$ are continuous functions with $g\left(0, x_{0}\right)=h\left(0, x_{0}\right)=0$. We assume further that the algebraic equation $z=f\left(0, x_{0}, x_{0}, z\right)$ has a real root $z_{0}$, and we require that

(2b) $x^{\prime}(0)=z_{0}$.

Existence and uniqueness theorems for IVP's for equation (1) have been proved by R. D. Driver [1] for the case where $h(t, x)<t$, and recently by J. K. Hale and M. A. Cruz [3] provided that $f$ is linear in the argument $x^{\prime}(h(t, x))$. We prove an existence theorem without these hypotheses, and a uniqueness theorem in case $h$ is independent of $x$. Hale and Cruz [3] have also obtained continuity theorems for the quasilinear case mentioned above, while Driver [2] has proved a continuity theorem for IVP's for equations of the form (1) in case $g$ and $h$ are both independent of $x$, and $h(t)<t$ for all $t$. We obtain here a continuous dependence result for the IVP (1)-(2a)-(2b) provided that the function $h$ is independent of $x$. Finally we obtain a result on existence of continuous solutions of certain nonlinear functional equations as a corollary of our existence and uniqueness theorems.

Received by the editors November 4, 1970.

AMS 1970 subject classifications. Primary 34K05; Secondary 34K05.

Key words and phrases. Neutral-differential equations, functional equations, continuous dependence, existence theory.

${ }^{1}$ Research supported by National Science Foundation Grant GP 20194.

Copyright (c) 1971, American Mathematical Society 
2. Existence. Let $\alpha>0$, and let $J=[-\alpha, \alpha]$. We shall make the following assumptions concerning the IVP (1)-(2a)-(2b):

(i) $f(t, x, y, z)$ is continuous in some region in $R^{4}$ containing $P=\left\{(t, x, y, z):|t| \leqq \alpha,\left|x-x_{0}\right| \leqq \beta,\left|y-x_{0}\right| \leqq \beta,|z| \leqq M\right\}$ where $\alpha, \beta$ and $M>\left|z_{0}\right|$ are positive constants. We assume that $\alpha \leqq \beta / M$ and that $\sup _{(t, x, y, z) \in P}|f(t, x, y, z)|<M$.

(ii) $g(t, x)$ and $h(t, x)$ are continuous in the projection $\tilde{R}$ of $P$ in the $(t, x)$ space; $g$ and $h$ both map $\widetilde{R}$ into $J$, with $g\left(0, x_{0}\right)=h\left(0, x_{0}\right)=0$, and $h(t, x)$ satisfies the Lipschitz conditions

$$
\left|h\left(t_{1}, x_{1}\right)-h\left(t_{2}, x_{2}\right)\right| \leqq k_{1}\left|t_{1}-t_{2}\right|+k_{2}\left|x_{1}-x_{2}\right|
$$

for all $\left(t_{1}, x_{1}\right),\left(t_{2}, x_{2}\right) \in R$, where $k_{1}$ and $k_{2}$ are nonnegative constants with $k_{1}+k_{2} M \leqq 1$.

(iii) The function $f(t, x, y, z)$ satisfies the Lipschitz condition

$$
\left|f\left(t, x, y, z_{1}\right)-f\left(t, x, y, z_{2}\right)\right| \leqq L_{z}\left|z_{1}-z_{2}\right|
$$

for all $\left(t, x, y, z_{1}\right),\left(t, x, y, z_{2}\right) \in P$, where $L_{z}<1$.

We shall prove the following theorem:

Theorem 1. Under the hypotheses (i)-(iii), the IVP (1)-(2a)-(2b) has at least one solution which is continuously differentiable on $J$.

Proof. Let $X$ be the Banach space of continuous functions on $J$ with uniform norm. Let

$$
S=\left\{z \in X: z(0)=z_{0},\|z\| \leqq M\right\} .
$$

Define the mapping $T: S \rightarrow S$ as follows: for $z \in S$, let

$$
T z(t)=f(t, I(z, t), I(z, g(t, I(z, t))), z(h(t, I(z, t)))),
$$

where

$$
I(z, t)=x_{0}+\int_{0}^{t} z(s) d s .
$$

It is easy to verify that $T$ is a continuous map of $S$ into $S$. By continuity of $f$, if $z \in S$ and $t \in J$, for each $\epsilon>0$, there exists $\delta(\epsilon)>0$ such that if $t_{1}$ and $t_{2} \in J$, and $\left|t_{1}-t_{2}\right|<\delta(\epsilon)$, then

$$
\begin{aligned}
\mid f\left(t_{1}, I\left(z, t_{1}\right), I\left(z, g\left(t_{1}, I\left(z, t_{1}\right)\right)\right), z(h(t, I(z, t)))\right) & \\
& -f\left(t_{2}, I\left(z, t_{2}\right), I\left(z, g\left(t_{2}, I\left(z, t_{2}\right)\right)\right), z(h(t, I(z, t)))\right) \mid<\epsilon .
\end{aligned}
$$

Let 


$$
\begin{aligned}
S_{e}=\left\{z \in S:\left|z\left(t_{1}\right)-z\left(t_{2}\right)\right|\right. & \leqq \epsilon /\left(1-L_{z}\right) \\
& \text { for all } \left.t_{1}, t_{2} \in J,\left|t_{1}-t_{2}\right| \leqq \delta(\epsilon)\right\} .
\end{aligned}
$$

If $z \in S_{\epsilon}$, and if $t_{1}, t_{2} \in J$ with $\left|t_{1}-t_{2}\right| \leqq \delta(\epsilon)$, then

$$
\left|T z\left(t_{1}\right)-T z\left(t_{2}\right)\right| \leqq \epsilon+\epsilon L_{z} /\left(1-L_{z}\right)=\epsilon /\left(1-L_{z}\right) .
$$

Thus $T S_{\boldsymbol{\epsilon}} \subset S_{\boldsymbol{\epsilon}}$. We note that $S_{\boldsymbol{\epsilon}}$ is closed, bounded and convex. Let $S_{0}=\bigcap_{\mathrm{all}}>>0 S_{\mathrm{e}} . S_{0}$ is nonempty, closed, bounded and convex, and by the Ascoli-Arzela theorem, $S_{0}$ is compact. Since $T S_{\epsilon} \subset S_{\mathrm{e}}$ for all $\epsilon>0, T S_{0} \subset S_{0}$. Hence by the Schauder theorem, $T$ has at least one fixed point $z(t)$. Integration yields the required solution of (1)-(2a)(2b).

3. Uniqueness. In case $h(t, x)$ is independent of $x$, we obtain the following uniqueness result:

THEOREM 2. In addition to the hypotheses of Theorem 1, suppose that:

(iv) $h(t, x) \equiv h(t)$ is independent of $x$.

(v) fand $g$ satisfy the Lipschitz conditions

$$
\begin{aligned}
& \left|f\left(t, x_{1}, y_{1}, z_{1}\right)-f\left(t, x_{2}, y_{2}, z_{2}\right)\right| \\
& \quad \leqq L\left\{\left|x_{1}-x_{2}\right|+\left|y_{1}-y_{2}\right|\right\}+L_{z}\left|z_{1}-z_{2}\right|
\end{aligned}
$$

with $L_{z}<1$;

$$
\left|g\left(t, x_{1}\right)-g\left(t, x_{2}\right)\right| \leqq L_{0}\left|x_{1}-x_{2}\right|,
$$

uniformly in their domains.

Then there exists $\gamma_{0}, 0<\gamma_{0} \leqq \alpha$, such that there is a unique continuously differentiable solution of the IVP (2)-(3a)-(3b) on $\left[-\gamma_{0}, \gamma_{0}\right]$.

Proof. Under the hypotheses of the theorem, if $z \in S, 0<\gamma \leqq \alpha$, and $t \in[-\gamma, \gamma]$,

$$
\begin{aligned}
\left|T z_{1}(t)-T z_{2}(t)\right| \leqq & L\left\{\left|I\left(z_{1}, t\right)-I\left(z_{2}, t\right)\right|\right. \\
& \left.\quad+\left|I\left(z_{1}, g\left(t, I\left(z_{1}, t\right)\right)\right)-I\left(z_{2}, g\left(t, I\left(z_{2}, t\right)\right)\right)\right|\right\} \\
& +L_{z}\left|z_{1}(h(t))-z_{2}(h(t))\right| \\
\leqq & L \gamma\left\|z_{1}-z_{2}\right\|+L \gamma\left\|z_{1}-z_{2}\right\| \\
& +L L_{g} M \gamma\left\|z_{1}-z_{2}\right\|+L_{z}\left\|z_{1}-z_{2}\right\| \\
= & {\left[\gamma L\left(2+M L_{o}\right)+L_{z}\right]\left\|z_{1}-z_{2}\right\| . }
\end{aligned}
$$

Hence if $\gamma$ is sufficiently small, the mapping $T$ is a contraction, and the statement of the theorem follows by integration. 
REMARK. A uniqueness theorem will follow also from the theorem in the next section.

4. Continuous dependence. For $i=1,2$, consider the IVP's

(1.i) $x_{i}^{\prime}(t)=f_{i}\left(t, x_{i}(t), x_{i}\left(g_{i}\left(t, x_{i}(t)\right)\right), x_{i}^{\prime}\left(h_{i}(t)\right)\right)$,

(2.ia) $x_{i}(0)=x_{i 0}$

(2.ib) $x_{i}^{\prime}(0)=z_{i 0}$

under hypotheses analogous to (i) $-(\mathrm{v})$ :

(H1) For $i=1,2, f_{i}(t, x, y, z)$ is continuous in some domain $D \subset R^{4}$ containing both of the sets

$P_{i}=\left\{(t, x, y, z):|t| \leqq a,\left|x-x_{i 0}\right| \leqq b,\left|y-x_{i 0}\right| \leqq b,|z| \leqq M\right\}$,

where $x_{i 0}$ are constants, $a, b$, and $M>\left|z_{i 0}\right|$ are constants with $\sup _{(t, x, y, z) \in D}\left|f_{i}(t, x, y, z)\right|<M$, and $z_{i 0}$ is a real root of the equation $z=f_{i}\left(t, x_{i 0}, x_{i 0}, z\right)$.

(H2) For $i=1,2, g_{i}(t, x)$ is continuous in the projection of $D$ in the $(t, x)$ plane, and $h_{i}(t)$ is continuous on $[-a, a]$, with $\left|g_{i}(t, x)\right| \leqq|t|$; $\left|h_{i}(t)\right| \leqq|t|$.

(H3) The functions $f_{1}$ and $g_{1}$ satisfy the conditions satisfied by $f$ and $g$ respectively in $\$ 3$.

TheOREM 3. Let (H1) $-(\mathrm{H} 3)$ be satisfied, let $\alpha=\min (a, b / M)$ and suppose that for $i=1,2, x_{i}(t)$ is a continuously differentiable function which satisfies (1.i) - (2.ia) - (2.ib), with

$$
\left|x_{10}-x_{20}\right|=\epsilon_{0}<\alpha M,
$$

and there exist nonnegative constants $\epsilon_{f}, \epsilon_{g}, \epsilon_{h}$ such that

$$
\begin{array}{r}
\left|f_{1}(t, x, y, z)-f_{2}(t, x, y, z)\right| \leqq \epsilon_{f} \\
\left|g_{1}(t, x)-g_{2}(t, x)\right| \leqq \epsilon_{o} \\
\left|h_{1}(t)-h_{2}(t)\right| \leqq \epsilon_{h}
\end{array}
$$

in their respective domains. Then if $\epsilon_{h}$ is sufficiently small, for all $t \in[-\alpha, \alpha]$,

$$
\left|x_{1}(t)-x_{2}(t)\right| \leqq \epsilon_{0}+C_{\epsilon, x_{1}}\left[\exp \left(\frac{\left(2+M L_{0}\right) L|t|}{1-L_{z}}\right)-1\right]
$$

where

$$
C_{e, x_{1}}=\frac{\epsilon_{f}+\left(2+M L_{0}\right) \epsilon_{0}+M L \epsilon_{g}+L_{z} \epsilon_{x_{1}, h}}{L\left(2+M L_{0}\right)}
$$

and for each fixed solution $x_{1}(t)$, the quantity $\epsilon_{x_{1}, h}$ tends to zero as $\epsilon_{h} \rightarrow 0$. 
Proof. Let $\eta>0$. By continuity of $x_{1}^{\prime}(t)$, there exists $\delta>0$ such that if $t, \tau \in[0, \alpha]$ and $|t-\tau|<\delta$, then $\left|x_{1}^{\prime}(t)-x_{1}^{\prime}(t)\right|<\eta$. We suppose that $\epsilon_{h}<\delta$. Set $z_{i}(t)=x_{i}^{\prime}(t), i=1,2$. The functions $z_{i}$ satisfy the equations

$$
\begin{aligned}
z_{i}(t)=f_{i}\left(t, x_{i 0}+\right. & \int_{0}^{t} z_{i}(s) d s, \\
& \left.x_{i 0}+\int_{0}^{g_{i}\left(t, x_{i 0}+\int_{0}^{t} z_{i}(\sigma) d \sigma\right)} z_{i}(s) d s, z_{i}\left(h_{i}(t)\right)\right) .
\end{aligned}
$$

Using the Lipschitz continuity of $f_{1}$, and the definitions of the quantities $\epsilon_{0}, \epsilon_{f}, \epsilon_{g}$ and $\eta$, we obtain from (4.1) and (4.2) the estimate

$$
\begin{aligned}
\left|z_{1}(t)-z_{2}(t)\right| \leqq \epsilon_{f} & +L\left\{\epsilon_{0}+\left|\int_{0}^{t}\right| z_{1}(s)-z_{2}(s)|d s|\right\} \\
& +L\left\{\epsilon_{0}+\left|\int_{0}^{g_{2}\left(t, x_{20}+\int_{0}^{t} z_{2}(\sigma) d \sigma\right)}\right| z_{1}(s)-z_{2}(s)|d s|\right. \\
& +\left|\int_{g_{2}\left(t, x_{20}+\int_{0}^{t} z_{2}(\sigma) d \sigma\right)}^{g_{1}\left(t, x_{20}+\int_{0}^{t} z_{2}(\sigma) d \sigma\right)}\right| z_{1}(s)|d s| \\
& \left.+\left|\int_{g_{1}\left(t, x_{20}+\int_{0}^{t} z_{2}(\sigma) d \sigma\right)}^{g_{1}\left(t, x_{10}+\int_{0}^{t} z_{1}(\sigma) d \sigma\right)}\right| z_{1}(s)|d s|\right\}
\end{aligned}
$$

The $a$ priori bound on $z_{1}(t)$ and the Lipschitz condition on $g_{1}(t, x)$, together with the fact that $\left|g_{2}(t, x)\right| \leqq|t|$, yield

$$
\begin{aligned}
& \left|z_{1}(t)-z_{2}(t)\right| \leqq \epsilon_{f}+\left(2+M L_{0}\right) L \epsilon_{0}+M L \epsilon_{o}+L_{z} \eta \\
& \quad+\left(1+M L_{0}\right) L\left|\int_{0}^{t}\right| z_{1}(s)-z_{2}(s)|d s| \\
& \quad+L \max \left\{\left|\int_{0}^{t}\right| z_{1}(s)-z_{2}(s)|d s|,\left|\int_{-t}^{0}\right| z_{1}(s)-z_{2}(s)|d s|\right\} \\
& \quad+L_{z}\left|z_{1}\left(h_{2}(t)\right)-z_{2}\left(h_{2}(t)\right)\right|
\end{aligned}
$$


Let $K=\epsilon_{f}+\left(2+M L_{o}\right) L \epsilon_{0}+M L \epsilon_{g}+L_{z} \eta$, and

$$
R(t)=\max _{|s| \leqq|t|}\left|z_{1}(s)-z_{2}(s)\right| .
$$

Then, on $[0, \alpha]$ we have

$$
R(t) \leqq K+\left(2+M L_{\vartheta}\right) L \int_{0}^{t} R(s) d s+L_{z} R\left(h_{2}(t)\right),
$$

and since $R$ is an even function, is nondecreasing, and $\left|h_{2}(t)\right| \leqq|t|$,

$$
R(t) \leqq \frac{K}{1-L_{z}}+\frac{\left(2+M L_{g}\right) L}{1-L_{z}} \int_{0}^{t} R(s) d s .
$$

By the Gronwall inequality

$$
R(t) \leqq \frac{K}{1-L_{z}} \exp \left(\frac{\left(2+M L_{g}\right) L t}{1-L_{z}}\right)
$$

and integration leads to

$$
\begin{aligned}
\left|x_{1}(t)-x_{2}(t)\right| & \leqq \epsilon_{0}+\int_{0}^{t} R(s) d s \\
& \leqq \epsilon_{0}+\frac{K}{\left(2+M L_{g}\right) L}\left[\exp \left(\frac{\left(2+M L_{g}\right) L t}{1-L_{z}}\right)-1\right],
\end{aligned}
$$

and setting $C_{\epsilon, x_{1}}=K /\left(2+M L_{\vartheta}\right) L$, we obtain (3) on $[0, \alpha]$. Since $R$ is an even function, the estimate (5) holds on $[-\alpha, 0]$ if $t$ is replaced by $-t$. Thus analogously the estimate (3) holds also on $[-\alpha, 0]$ and the proof is complete.

5. Nonlinear functional equations. As a corollary to our existence and uniqueness results, we note that if $f(t, x, y, z)$ is independent of $x$ and $y$, and $h(t, x)$ is independent of $x$, the problem $(1)-(2 b)$ has the form of the functional equation

(5) $z(t)=f(t, z(h(t)))$,

(6) $z(0)=z_{0}$,

where $z_{0}$ is a root of $z=f(0, z)$. Theorems 1 and 2 then yield at once:

THEOREM 4. Let $f(t, z)$ be continuous in some region in $R^{2}$ containing $P_{1}=\{t:|t| \leqq \alpha,|z| \leqq M\}$, where $\alpha$ and $M$ are positive constants such that $\sup _{(t, z) \in P_{1}}|f(t, z)|<M$, and $M>\left|z_{0}\right|$ where $z_{0}$ is a real root of $\boldsymbol{z}=f(0, z)$. Let $f$ satisfy the Lipschitz condition $\left|f\left(t, z_{1}\right)-f\left(t, z_{2}\right)\right|$ $\leqq L_{z}\left|z_{1}-z_{2}\right|$ for all $\left(t, z_{1}\right),\left(t, z_{2}\right) \in P_{1}$, with $L_{z}<1$. Let $h(t)$ be continuous for $|t| \leqq \alpha, h(0)=0$, and $\left|h\left(t_{1}\right)-h\left(t_{2}\right)\right| \leqq\left|t_{1}-t_{2}\right|$ for $t_{1}, t_{2} \in[-\alpha, \alpha]$. 
The the problem (5)-(6) has at least one continuous solution on $[-\alpha, \alpha]$, and this is the unique continuous solution on this interval if $\alpha$ is sufficiently small.

\section{REFERENCES}

1. R. D. Driver, A functional-differential system of neutral type arising in a twobody problem of classical electrodynamics, Internat. Sympos. on Nonlinear Differential Equations and Nonlinear Mechanics, Academic Press, New York, 1963, pp. 474-484. MR 26 \#4008.

2. - Existence and continuous dependence of solutions of a neutral functionaldifferential equation, Arch. Rational Mech. Anal. 19 (1965), 149-166. MR 31 \#3654.

3. J. K. Hale and M. A. Cruz, Existence, uniqueness, and continuous dependence for hereditary systems, Ann. Mat. Pura Appl. 85 (1970), 63-81.

University of Missouri-Rolla, Rolla, Missouri 65401 